\title{
On some topics in the theory of infinite dimensional linear groups
}

\author{
M. R. Dixon, L. A. Kurdachenko, N. N. Semko, \\ and I. Ya. Subbotin
}

\begin{abstract}
A B S TRACT. In this paper we present a synopsis of some recent results concerned with infinite dimensional liner groups, including generalizations of irreducibility, the central dimension of a linear group, groups with finite dimensional orbits and the maximal and minimal conditions on subgroups of infinite central dimension.
\end{abstract}

\section{Introduction}

Linear groups are groups of invertibile linear transformations of vector spaces and are among the most important mathematical objects. Due to their versatility and penetration into areas quite different from each other, their imprint can be found in almost all mathematical (and not only mathematical) disciplines. Their connections with other disciplines, including natural sciences, are very diverse and nontrivial. At present, it is quite difficult to imagine areas of natural science where vector spaces along with their transformations do not appear. Linear groups appeared in the form of geometric transformations in F. Klein's Erlangen Program and were also one of the sources for the emergence of the general theory of groups. The theory of linear groups is one of the oldest and most developed theories of modern mathematics. The utility of the matrix apparatus made it possible for a very deep development of the theory of finite dimensional linear groups. In essence the theory of finite dimensional linear groups is

2010 MSC: Primary 20H25; Secondary 20F29, 20E34.

Key words and phrases: finite central dimension, irreducible group, quasiirreducible group, FC-hypercentral, augmentation dimension, finite orbit, finite dimensional orbit, minimal condition, maximal condition. 
the theory of matrix groups. There is a vast array of articles and books devoted to the study of finite dimensional linear groups, far too numerous to mention. Many interesting results were obtained, which contributed to progress both in the general theory of groups and in many other fields.

The theory of infinite dimensional linear groups is fundamentally different from the theory of finite dimensional linear groups. As for finite dimensional linear groups, one can consider matrices of linear transformations with respect to various bases. These matrices will be infinite, but have a finiteness property, since only a finite number of basis elements are involved in the decomposition of each vector space element; then each column of such an infinite matrix has only a finite set of nonzero coefficients. However, the theory of infinite matrices is far from being as well established as the theory of matrices of finite size, although the beginnings of this theory can be traced back to the 19th century. Infinite matrices and determinants were introduced into analysis by Poincaré in 1884 in the discussion of the well-known Hill's equation. The rapid development of the theory of linear spaces of infinite dimension began at the beginning of the 20th century. The foundations were laid mainly by the studies of Ivar Fredholm and Vito Volterra. They considered the theory of linear equations with an infinite number of equations and unknowns using the limit representation of linear equations with a finite number of equations and unknowns when the number of equations and unknowns becomes infinite. This led to the development of the theory of integral equations. On the other hand, the works of David Hilbert, John von Neumann, Erhard Schmidt and Friges Riesz on the theory of integral equations gave impetus to the development of the theory of linear spaces of infinite dimension. This led to the creation of the theory of Banach and Hilbert spaces. Some overview of this topic can be found in the book of R. G. Cooke [3] and the article of M. Bernkopf [2]. However, an exhaustive search of all these areas shows that the main object of study was infinite matrices over the fields of real and complex numbers, and this study was carried out using the very well established apparatus of mathematical analysis. A study of the algebraic properties of groups of infinite matrices over arbitrary fields began relatively recently (see the book of W. Holubowski [21]). In the bibliography we give some works related to this topic. We have a similar situation with infinite dimensional linear groups.

In this paper we give some of the recent activity concerned with the algebraic aspects of infinite dimensional linear groups. In Section 2 we show some of the main differences between finite dimensional and infinite dimensional linear groups. We define Kurosh-Chernikov systems and show 
how these can impact the structure of our group, giving the main types of system that can occur. In Section 3 we discuss quasi-irreducible and related groups. These are generalizations of irreducible groups. We also discuss almost irreducible groups which in some sense are dual to the quasiirreducible groups. In Section 4 we introduce the central dimension and augmentation dimension of a linear group. These dimensions are intimately connected in a manner that is analogous to way the central quotient group and derived subgroup of a group are connected. In Section 5 we look at groups with various finiteness conditions on the size of the orbits of an element of the vector space $A$. Finally in Section 6 we consider infinite dimensional linear groups with the maximal (or minimal) conditions on subgroups of infinite central dimension.

Our notation where not explained is generally standard.

\section{Kurosh-Chernikov systems}

There has been significant progress in the theory of linear groups, not for groups of arbitrary linear transformations, but for those that have some additional properties that make it possible to use the tools from analysis. For fields that are not endowed with topological structures the situation is different and the difference from finite dimensional linear groups is evident from the beginning. If $A$ is a vector space over a field $F$ on which a group $G$ of linear transformations acts and if $A$ has finite dimension, then $A$ has a finite series of $G$-invariant subspaces

$$
0=A_{0} \leqslant A_{1} \leqslant \cdots \leqslant A_{n}=A
$$

whose factors are $G$-chief. The factors $G / C_{G}\left(A_{j} / A_{j-1}\right)$ are irreducible linear groups when $1 \leqslant j \leqslant n$ and the stabilizer $\bigcap_{1 \leqslant j \leqslant n} C_{G}\left(A_{j} / A_{j-1}\right)$ of this series is a nilpotent subgroup. When the vector space $A$ has infinite dimension, then $A$ is not guaranteed to have a finite $G$-chief series. However, certain natural families of $G$-invariant subspaces can be studied.

Let $F$ be a field and let $A$ be a vector space over $F$. The $\operatorname{set} \operatorname{End}(F, A)$ of all linear transformations of $A$ is an associative $F$-algebra with identity in which the operations are addition, composition of linear transformations and scalar multiplication by elements of the field $F$. The group $G L(F, A)$ of all invertible linear transformations (non-singular transformations or $F$-automorphisms) of $A$ is called the General Linear Group of $A$. The subgroups of $G L(F, A)$ are called linear groups.

Let $G \leqslant G L(F, A)$ and let $C, D$ be $G$-invariant subspaces of $A$ such that $D \leqslant C$. Then $C / D$ is called a $G$-chief factor if, whenever $B$ is a $G$ - 
invariant subspace such that $D \leqslant B \leqslant C$, it follows that either $B=D$ or $B=C$. When we think of $A$ as an $F G$-module, this means that a $G$-chief factor $C / D$ is a simple $F G$-module.

A subgroup $G$ of $G L(F, A)$ is called irreducible if $A$ contains no proper non-zero $G$-invariant subspaces. In this case $A$ is $G$-chief.

Let $\mathcal{S}$ be a family of $G$-invariant subspaces of $A$, linearly ordered by inclusion. Then $\mathcal{S}$ is called a complete system if it satisfies the condition:

- For every subfamily $\mathcal{L}$ of $\mathcal{S}$ the intersection and union of all members of $\mathcal{L}$ belong to $\mathcal{S}$.

It is possible to prove that every family of $G$-invariant subspaces of $A$, linearly ordered by inclusion, can be extended to a complete system.

Let $\mathcal{S}$ be a family of $G$ invariant subspaces of $A$. Then a pair $(B, C) \in \mathcal{S}$, where $B \leqslant C$, is a jump of $\mathcal{S}$ if it satisfies the condition:

- For every element $D \in \mathcal{S}$ such that $B \leqslant D \leqslant C$, either $B=D$ or $D=C$.

If the pair $(B, C)$ is a jump of $\mathcal{S}$, then $C / B$ is called a factor of the system $\mathcal{S}$.

Suppose now that $\mathcal{S}$ is a complete system of $G$-invariant subspaces of $A$. For each $0 \neq a \in A$ let

$$
V_{a}=\bigcup\{V \in \mathcal{S} \mid a \notin V\} \quad \text { and } \quad \Lambda_{a}=\bigcap\{V \in \mathcal{S} \mid a \in V\}
$$

Since $\mathcal{S}$ is a complete system it follows that $V_{a}, \Lambda_{a} \in \mathcal{S}$ and since $\mathcal{S}$ is a linearly ordered system, we must have $V_{a} \leqslant \Lambda_{a}$ and it is then easy to see that the pair $\left(V_{a}, \Lambda_{a}\right)$ is a jump of the system $\mathcal{S}$. Conversely, let $(B, C)$ be a jump of the system $\mathcal{S}$. Choose $y \in C \backslash B$. From the definitions of $V_{y}$ and $\Lambda_{y}$ we see that $B \leqslant V_{y}$ and $\Lambda_{y} \leqslant C$. Since $y \in C$ it follows that $V_{y} \neq C$ and since $(B, C)$ is a jump, we deduce that $B=V_{y}$. Also, since $\Lambda_{y} \neq B$ and $(B, C)$ is a jump, we deduce that $\Lambda_{y}=C$.

When $A$ is a vector space over the field $F$ and $G$ is a subgroup of $G L(F, A)$, a family $\mathcal{S}$ of $G$-invariant subspaces of $A$ is called a KuroshChernikov system, if it satisfies the conditions

(KC1) $0, A \in \mathcal{S}$;

$(\mathrm{KC} 2) \mathcal{S}$ is linearly ordered and complete.

Families of this kind were originally introduced in the classical article of A. G. Kurosh and S. N. Chernikov [40] concerning groups with operators. We observe that vector spaces are examples of groups with operators.

If $\mathcal{S}, \mathcal{R}$ are families of $G$-invariant subgroups, linearly ordered by inclusion, then we say that $\mathcal{R}$ is a refinement of $\mathcal{S}$ if every term of $\mathcal{S}$ is also a term of $\mathcal{R}$ (thus, $\mathcal{S} \subseteq \mathcal{R}$ ). If $\mathcal{S}$ is a proper subset of $\mathcal{R}$, then $\mathcal{R}$ is called 
a proper refinement of $\mathcal{S}$. If $\mathcal{S}$ is a Kurosh-Chernikov system, then $\mathcal{S}$ is $G$-chief if it has no proper refinement and it is possible to prove that a Kurosh-Chernikov system is $G$-chief if and only if every one of its factors is $G$-chief.

Furthermore, it is also possible to prove that every family of $G$-invariant subspaces of $A$, linearly ordered by inclusion, can be extended to a $G$-chief Kurosh-Chernikov system.

There are many important types of Kurosh-Chernikov systems $\mathcal{S}$. For example, suppose first that $\mathcal{S}$ is a totally ordered ascending family of subspaces.

We can construct an ascending series

$$
\begin{aligned}
0 & =A_{0} \leqslant A_{1} \leqslant \ldots \leqslant A_{n} \leqslant A_{n+1} \leqslant \ldots A_{\omega} \leqslant A_{\omega+1} \leqslant \ldots \\
& \leqslant A_{\alpha} \leqslant A_{\alpha+1} \leqslant \ldots A_{\gamma}=A,
\end{aligned}
$$

where for limit ordinals $\nu$ we have $\bigcup_{\beta<\nu} A_{\beta}=A_{\nu}$.

On the other hand, if a Kurosh-Chernikov system $\mathcal{S}$ is a descending totally ordered system, then we obtain in similar fashion a descending series

$$
\begin{aligned}
A & =A_{0} \geqslant A_{1} \geqslant \ldots \geqslant A_{n} \geqslant A_{n+1} \geqslant \ldots A_{\omega} \geqslant A_{\omega+1} \\
& \geqslant \ldots A_{\alpha} \geqslant A_{\alpha+1} \geqslant \ldots A_{\gamma}=0
\end{aligned}
$$

where for limit ordinals $\nu$ we now have $A_{\nu}=\bigcap_{\beta<\nu} A_{\beta}$.

A Kurosh-Chernikov system $\mathcal{S}$ is called $G$-central, if every factor is $G$-central in the usual way.

A vector space $A$ is called $G$-hypercentral if it has an ascending $G$ central series of $G$-invariant subspaces terminating in $A$ and $A$ is called $G$-nilpotent if this ascending series has finite length. In the latter case it is possible to prove that the group $G$ is nilpotent. However, if $A$ has infinite dimension and is $G$-hypercentral, then $G$ may contain free groups or certain types of exotic groups, so that in this case $G$ may be very far from being nilpotent.

Now let $\mathcal{S}$ be a Kurosh-Chernikov system and let $\mathcal{T}$ be the set of all jumps of $\mathcal{S}$. For each jump $(B, C)$ of $\mathcal{S}$ and each $g \in G$, let $[g, C]=$ $\langle g(c)-c \mid c \in C\rangle$, let $C_{G}(C / B)=\{g \in G \mid[g, C] \leqslant B\}$ and define

$$
\operatorname{St}_{G}(\mathcal{S})=\bigcap_{(B, C) \in \mathcal{T}} C_{G}(C / B) .
$$

This subgroup $\operatorname{St}_{G}(\mathcal{S})$ is called the stability group of the system $\mathcal{S}$ or the stabilizer of $\mathcal{S}$. With this definition, $\mathcal{S}$ is a $\operatorname{St}_{G}(\mathcal{S})$-central system and conversely, if a Kurosh-Chernikov system is $G$-central, then $G=\operatorname{St}_{G}(\mathcal{S})$. 
As in the finite dimensional case, Remak's theorem implies the embedding of $G / \operatorname{St}_{G}(\mathcal{S})$ into the Cartesian product $\underset{(B, C) \in \mathcal{T}}{\mathrm{Cr}} G / C_{G}(C / B)$. In particular, if $\mathcal{S}$ is $G$-chief, then each of the factor groups $G / C_{G}(C / B)$ is irreducible. However there are many differences with the finite dimensional case here.

The most obvious difference is that in general the set of components in the Cartesian product will be infinite.

In this case, we are no longer dealing with subgroups of a direct product, but with subgroups of a Cartesian product. This alone makes the task of studying such groups much more complicated.

The situation with the subgroup $\operatorname{St}_{G}(\mathcal{S})$ is also much more complicated here. In the case when $A$ has infinite dimension, $\operatorname{St}_{G}(\mathcal{S})$ may be very far from being nilpotent.

\section{Quasi-irreducible and almost irreducible groups}

As we noted above every factor $V / U$ of the $G$-chief Kurosh-Chernikov system $\mathcal{S}$ is $G$-chief. Then $G / C_{G}(V / U)$ is an irreducible linear subgroup of $G L(F, V / U)$. In this way we obtain one of the important types of linear groups namely irreducible linear groups. For infinite dimensional irreducible linear groups the situation is also significantly different from the finite dimensional case where the use of powerful techniques from matrix theory is not always possible. Consequently the description of infinite dimensional irreducible linear groups must be obtained under stronger restrictions than in the finite dimensional case.

Chapters 2, 3 of the book [32] contain fairly detailed information about these groups, so we shall not dwell on them, but consider in more detail some linear groups that are close to irreducible in one way or another.

Let $A$ be a vector space over the field $F$ and let $G$ be a subgroup of $G L(F, A)$. If the group $G$ is irreducible, then every proper $G$-invariant subspace of $A$ is trivial and, in particular, has finite dimension. Thus a linear group $G$ whose action on the (infinite dimensional) vector space $A$ has the property

- every proper $G$-invariant subspace of $A$ has finite dimension can be considered as a generalization of an irreducible linear group. There are two situations that arise here for an infinite dimensional linear group $G$ :

(i) Every proper $G$-invariant subspace of $A$ has finite dimension and $A$ is the ascending union of certain proper $G$-invariant subspaces, or 
(ii) $A$ contains a proper $G$-invariant finite dimensional subspace $B$ such that the factor $A / B$ is $G$-chief.

A linear group $G$ that satisfies condition (i) is said to be quasiirreducible.

The study of quasi-irreducible linear groups was begun by D. I. Zaitsev in the paper [53] for the case when $F$ is a finite field. The case when $F$ is an arbitrary field was considered in the papers of L. A. Kurdachenko and I. Ya. Subbotin $[35,36]$. In turn these results were generalized by L. A. Kurdachenko [24] to the case of modules over group rings $R G$ when $R$ is a Dedekind domain. We shall now discuss the structure of quasi-irreducible linear groups as determined in the articles [24,35,36]. We also mention here the papers $[23,34]$.

When $F$ is a field, $A$ is a vector space over $F$ and $G$ is a subgroup of $G L(F, A)$, then let $\mathfrak{M}$ be the family of all minimal $G$-invariant subspaces of $A$. We let $\operatorname{Soc}_{G}(A)$ denote the subspace generated by all subspaces in the family $\mathfrak{M}$. One can prove that $\operatorname{Soc}_{G}(A)=\bigoplus_{\lambda \in \Lambda} A_{\lambda}$ where $A_{\lambda}$ is a minimal $G$-invariant subspace for each $\lambda \in \Lambda$. The subspace $\operatorname{Soc}_{G}(A)$ is called the $G$-socle of the space $A$.

Let $R$ be an integral domain and let $A$ be an $R$-module. Let

$$
\operatorname{Tor}_{R}(A)=\left\{a \in A \mid \operatorname{Ann}_{R}(a) \neq 0\right\} .
$$

Then $\operatorname{Tor}_{R}(A)$ is an $R$-submodule of $A$ called the $R$-periodic part of $A$. We say that $A$ is periodic as an $R$-module or, simply, that $A$ is $R$-periodic, if $\operatorname{Tor}_{R}(A)=A$. We say that $A$ is $R$-torsion-free if $\operatorname{Tor}_{R}(A)=0$.

We define the $R$-assassinator of $A$ to be the set

$\operatorname{Ass}_{R}(A)=\left\{P \mid P\right.$ is a prime ideal of $R$ such that $\left.\operatorname{Ann}_{A}(P) \neq 0\right\}$.

If $U$ is an ideal of $R$, then we set

$$
A_{U}=\left\{a \in A \mid U^{n} a=0 \text { for some natural number } n\right\}
$$

It is easy to see that $A_{U}$ is an $R$-submodule of $A$ called the $U$-component of $A$. If $A=A_{U}$, then $A$ is called a $U$-module. Furthermore, let

$$
\Omega_{U, k}(A)=\left\{a \in A \mid U^{k} a=0 \text { for this fixed } k\right\} .
$$

Then $\Omega_{U, k}(A)$ is an $R$-submodule of $A$. Also

$$
\Omega_{U, 1}(A) \leqslant \Omega_{U, 2}(A) \leqslant \cdots \leqslant \Omega_{U, k}(A) \leqslant \cdots \leqslant A_{U}
$$


and

$$
A_{U}=\bigcup_{k \in \mathbb{N}} \Omega_{U, k}(A)
$$

Let $D$ be a Dedekind domain and let $A$ be a simple $D$-module. Then $A \cong D / P$ for some maximal ideal $P$. We note that $D / P^{k}$ and $P / P^{k+1}$ are isomorphic as $D$-modules, for all $k \in \mathbb{N}$ (see [33, Corollary 1.28], for example). In particular, $D / P^{k}$ is embedded in $D / P^{k+1}$ for each $k \in$ $\mathbb{N}$. Hence we may form the injective limit of the family of $D$-modules $\left\{D / P^{k} \mid k \in \mathbb{N}\right\}$ which we denote by $C_{P^{\infty}}$.

The $D$-module $C_{P \infty}$ is called a Prüfer $P$-module.

It follows from its construction that $C_{P \infty}$ is a $P$-module and moreover

$$
\boldsymbol{\Omega}_{P, k}\left(C_{P \infty}\right) \cong_{D} D / P^{k} \text { for all } k \in \mathbb{N} .
$$

Furthermore,

$$
\boldsymbol{\Omega}_{P, k+1}\left(C_{P \infty}\right) / \boldsymbol{\Omega}_{P, k}\left(C_{P^{\infty}}\right) \cong\left(D / P^{k+1}\right) /\left(P / P^{k+1}\right) \cong D / P .
$$

Also, if $C$ is a proper $D$-submodule of $C_{P \infty}$, then there exists a natural number $k$ such that $C=\boldsymbol{\Omega}_{P, k}\left(C_{P \infty}\right)$. Similarly, if $b \notin \boldsymbol{\Omega}_{P, k-1}\left(C_{P \infty}\right)$, then $C=b D$.

We also observe that a Prüfer $P$-module is monolithic and its monolith coincides with $\boldsymbol{\Omega}_{P, 1}\left(C_{P \infty}\right)$.

Let $G$ be a group and $\pi$ be some set of primes. If $\mathcal{S}$ is a family of periodic normal $\pi$-subgroups of $G$, then clearly the subgroup generated by all subgroups in the family $\mathcal{S}$ is also a $\pi$-group. It follows that every group $G$ has a greatest normal $\pi$-subgroup which we denote by $\mathbf{O}_{\pi}(G)$.

The following results hold for quasi-irreducible modules.

Theorem 1. Let $A$ be a vector space over the field $F$ and let $G$ be a locally radical quasi-irreducible subgroup of $G L(F, A)$. If $C_{G}\left(\boldsymbol{S o c}_{G}(A)\right)=1$, then the following assertions hold:

(i) G contains a normal abelian subgroup $U$ of finite index;

(ii) the torsion subgroup $\operatorname{Tor}(U)$ of the subgroup $U$ has finite special rank;

(iii) if $\operatorname{char}(F)=p>0$, then $O_{p}(G)=1$;

(iv) there is a $U$-invariant subspace $B$ of $A$ such that $U$ is a quasiirreducible subgroup of $G L(F, B)$;

(v) $U$ contains an element $x$ of infinite order such that $B$ is $P$-periodic for some maximal ideal $P$ of the ring $F\langle x\rangle$. Moreover,

$$
B=C_{1} \oplus \cdots \oplus C_{n}
$$

where $C_{j}$ is a Prüfer P-module, for $1 \leqslant j \leqslant n$; 
(vi) $A=B \oplus g_{1} B \oplus \cdots \oplus g_{t} B$, where $\left\{1, g_{1}, \ldots, g_{t}\right\}$ is a transversal to $U$ in $G$.

When $C_{G}\left(\operatorname{Soc}_{G}(A)\right) \neq 1$ we have:

Theorem 2. Let $A$ be a vector space over the field $F$ and let $G$ be a hypercentral quasi-irreducible subgroup of $G L(F, A)$. If $C_{G}\left(\boldsymbol{S o c}_{G}(A)\right) \neq 1$ then:

(i) $G$ is abelian-by-finite;

(ii) the torsion subgroup $\operatorname{Tor}(G)$ has finite special rank;

(iii) if $\operatorname{char}(F)=p$ is a prime, then $\operatorname{Tor}(G)$ is a $p^{\prime}$-group;

(iv) $\operatorname{Tor}(G) \cap \zeta(G)$ is locally cyclic;

(v) $C_{G}\left(\boldsymbol{S o c}_{G}(A)\right) \cap \zeta(G)$ contains an element $x$ of infinite order such that $A$ is $F\langle x\rangle$-periodic and $\boldsymbol{A s s}_{F\langle x\rangle}(A)=\{P\}$ where $P$ is the ideal of $F\langle x\rangle$ generated by $x-1$;

(vi) $A=C_{1} \oplus \cdots \oplus C_{n}$ where $C_{j}$ is a Prüfer P-module for $1 \leqslant j \leqslant n$.

We now consider the second of the situations that arose earlier and suppose that $A$ contains a proper finite dimensional $G$-invariant subspace $B$ such that the factor $A / B$ is $G$-chief. This situation was considered in the paper [16] and we now give a description of the following main result of this work.

For the group $G$ if $x \in G$, then let

$$
x^{G}=\left\{x^{g}=g^{-1} x g \mid g \in G\right\} \quad \text { and } \quad \mathbf{F C}(G)=\left\{x \in G \mid x^{G} \text { is finite }\right\},
$$

this latter characteristic subgroup of $G$ being the $F C$-center of $G$.

Beginning with the FC-center we may construct the upper FC-central series of the group $G$, the series of characteristic subgroups of $G$,

$$
1=C_{0} \leqslant C_{1} \leqslant \ldots C_{\alpha} \leqslant C_{\alpha+1} \leqslant \ldots C_{\gamma},
$$

defined by

$$
\begin{aligned}
C_{1} & =\mathbf{F C}(G), \\
C_{\alpha+1} / C_{\alpha} & =\mathbf{F C}\left(G / C_{\alpha}\right) \text { for ordinals } \alpha, \\
C_{\lambda} & =\bigcup_{\beta<\lambda} C_{\beta} \text { for limit ordinals } \lambda .
\end{aligned}
$$

The last term $C_{\gamma}$ of this series is called the upper FC-hypercenter of the group $G$ and if $G=C_{\gamma}$, then $G$ is called FC-hypercentral. 
We note that if $x \in \mathbf{F C}(G)$ has finite order, then the normal closure $\langle x\rangle^{G}$ is finite (see [17, Proposition 1.5.2] for example). Thus if $H$ is a $G$ invariant periodic subgroup of $\mathbf{F C}(G)$, then $H$ has an ascending series of $G$-invariant subgroups whose factors are finite. This implies that if $H$ is a $G$-invariant periodic subgroup of the upper FC-hypercenter of $G$, then $H$ has an ascending series of $G$-invariant subgroups whose factors are finite.

The main result of [16] is

Theorem 3. Let $A$ be a vector space over $F$ and let $G$ be an $F C$ hypercentral subgroup of $G L(F, A)$. Let $A$ have a finite $F G$-composition series. Then $A$ contains two $F G$-submodules $B, C$ satisfying the following conditions:

(i) each $G$-chief factor of $B$ has finite F-dimension;

(ii) each $G$-chief factor of $C$ has infinite $F$-dimension;

(iii) $A=B \oplus C$.

Corollary 1. Let $A$ be a vector space over $F$ and let $G$ be an $F C$ hypercentral subgroup of $G L(F, A)$. Suppose that $A$ contains a finite dimensional $G$-invariant subspace $B$ such that $A / B$ is infinite dimensional and $G$-chief. Then $A$ contains a $G$-invariant subspace $C$ such that $A=B \oplus C$.

Corollary 2. Let $A$ be a vector space of infinite dimension over $F$ and let $G$ be an FC-hypercentral group of $G L(F, A)$. If every proper $G$-invariant subspace of $A$ has finite $F$-dimension, then either $G$ is irreducible or $G$ is quasi-irreducible.

In this regard we note that Corollary 2 is no longer true even for the case when $G$ is soluble. A corresponding counterexample was constructed in the paper [52] of D. I. Zaitsev.

Let $F$ be a field, $A$ a vector space over $F$ and let $G$ be a subgroup of $G L(F, A)$. If the group $G$ is irreducible, then every non-zero $G$-invariant subspace of $A$ coincides with $A$. In particular every non-zero $G$-invariant subspace has finite codimension. So the linear groups whose action on the vector space is determined by the following property

every non-zero $G$-invariant subspace of $A$ has finite codimension.

can be considered as a generalization of irreducible linear groups. The following two situations can arise here:

(i) every non-zero $G$-invariant subspace of $A$ has finite codimension and the intersection of all the non-zero $G$-invariant subspaces is zero;

(ii) $A$ contains a minimal non-zero $G$-invariant subspace $B$ of finite codimension. 
If a linear group $G$ satisfies condition (i) above then the linear group $G$ will be called almost irreducible.

From Theorem 3 we obtain

Corollary 3. Let $A$ be a vector space of infinite dimension over $F$ and let $G$ be an $F C$-hypercentral subgroup of $G L(F, A)$. If every non-zero $G$ invariant subspace of $A$ has finite codimension over $F$, then either $G$ is irreducible or an almost irreducible subgroup of $G L(F, A)$.

Almost irreducible infinite dimensional linear groups were studied in the paper [37] of L. A. Kurdachenko and I. Ya. Subbotin (see also the survey paper [23]). In the paper [34] of L. A. Kurdachenko and I. Ya. Subbotin these results were generalized to the case of modules over a group ring $R G$ where $R$ is a Dedekind domain. Here we give a description of the structure of almost irreducible linear groups found in [37].

We recall that a group $G$ has finite special rank $r(G)=r$ if every finitely generated subgroup of $G$ can be generated by $r$ elements and $r$ is the least positive integer with this property. If there is no such integer $r$ then $G$ is said to be of infinite special rank. The general concept of special rank (and also the term "special rank") was introduced by A. I. Maltsev [41]. There is an extensive literature concerned with groups of special rank and essential information associated with groups of finite special rank can be found in [17].

Theorem 4. Let $A$ be a vector space over the field $F$ and let $G$ be an almost irreducible hypercentral subgroup of $G L(F, A)$. Then $G$ satisfies the following conditions:

(i) $G$ is abelian-by-finite;

(ii) the torsion subgroup $T$ of $G$ has finite special rank;

(iii) $T \cap \zeta(G)$ is a locally cyclic group;

(iv) if the field $F$ has prime characteristic $p$, then $T$ is a $p^{\prime}$-subgroup.

This theorem allows the following generalization.

Theorem 5. Let $A$ be a vector space over the field $F$ and let $G$ be an almost irreducible locally soluble $F C$-hypercentral subgroup of $G L(F, A)$. Then $G$ satisfies the following conditions:

(i) $G$ is abelian-by-finite;

(ii) the torsion subgroup $T$ of the group $G$ has finite special rank;

(iii) $T \cap \zeta(G)$ is a locally cyclic group;

(iv) if the field $F$ has prime characteristic $p$, then $T$ is a $p^{\prime}$-subgroup. 


\section{Finite central dimension}

We now consider linear groups that are, in some sense, close to finite dimensional.

If $G$ is a subgroup of $G L(F, A)$ then let $Z=\zeta_{G}(A)=C_{A}(G)$. Then as we have seen above $\zeta_{G}(A)$ is a $G$-invariant subspace called the $G$-center of $A$ and $G$ acts trivially on $Z$. Thus we see that actually $G$ acts on the quotient space $A / Z$.

We say that the central dimension of $G$ is the dimension of the quotient space $A / \zeta_{G}(A)$. We denote the central dimension of the linear group $G$ by $\operatorname{centdim}_{F}(G)$.

In particular, if $\operatorname{centdim}_{F}(G)=\operatorname{dim}_{F}\left(A / \zeta_{G}(A)\right)$ is finite, then we shall say that $G$ has finite central dimension.

We immediately note the following properties of groups having finite central dimension.

1) If $G$ has finite central dimension and $\operatorname{char}(F)$ is the prime $p$, then $G$ contains an elementary abelian normal $p$-subgroup $L$ such that $G / L$ is isomorphic to some subgroup of $G L_{n}(F)$, where $n=$ $\operatorname{centdim}_{F}(G)$.

2) If $G$ has finite central dimension and $\operatorname{char}(F)=0$, then $G$ contains a torsion-free abelian normal subgroup $L$ such that $G / L$ is isomorphic to some subgroup of $G L_{n}(F)$, where $n=\operatorname{centdim}_{F}(G)$.

An element $g \in G L(F, A)$ is called finitary if $\operatorname{dim}_{F}\left(A / C_{A}(g)\right)$ is finite. In particular, this means that the cyclic group $\langle g\rangle$ has finite central dimension in this case. A subgroup $G$ of $G L(F, A)$ is called finitary if each element of $G$ is finitary. It is easy to see that a group $G$ is finitary if and only if every finitely generated subgroup of $G$ has finite central dimension.

The isomorphism $[g, A] \cong A / C_{A}(g)$ implies that $\operatorname{dim}_{F}\left(A / C_{A}(g)\right)=$ $\operatorname{dim}_{F}([g, A])$. Thus the element $g$ is finitary if and only if $\operatorname{dim}_{F}([g, A])$ is finite. In general, if $G$ is an arbitrary subgroup of $G L(F, A)$, then $G$ acts trivially on $A /[G, A]$ so that $G$ really acts on $[G, A]$. We recall that $[G, A]$ is the subspace generated by all elements of the form $(g-1) a$, for all $a \in A$ and $g \in G$. Considering $A$ as a module for the group $\operatorname{ring} F G$, then the subring $\omega(F G)$ generated by all elements of the form $g-1$, for $g \in G$ is a two sided ideal of $F G$ called the augmentation ideal of $F G$. Using this approach $[G, A]$ is then the $F G$-submodule $\omega(F G) A$.

We define the augmentation dimension of a group $G$ to be the dimension of the subspace $[G, A]$ and we denote this by $\operatorname{augdim}_{F}(G)$.

In particular, if $\operatorname{augdim}_{F}(G)=\operatorname{dim}_{F}([G, A])$ is finite, then we shall say that the group $G$ has finite augmentation dimension. 
The properties of the augmentation dimension are similar to those of the central dimension. In particular:

1) If $G$ has finite augmentation dimension and $\operatorname{char}(F)=p$ is prime, then $G$ contains an elementary abelian normal $p$-subgroup $L$ such that $G / L$ is isomorphic to some subgroup of $G L_{n}(F)$, where $n=$ $\operatorname{augdim}_{F}(G)$.

2) If $G$ has finite augmentation dimension and $\operatorname{char}(F)=0$, then $G$ contains a torsion-free abelian normal subgroup $L$ such that $G / L$ is isomorphic to some subgroup of $G L_{n}(F)$, where $n=\operatorname{augdim}_{F}(G)$.

We note that if $G$ is a finitely generated subgroup of $G L(F, A)$, then $\operatorname{centdim}_{F}(G)$ is finite if and only if $\operatorname{augdim}_{F}(G)$ is finite. However, for arbitrary subgroups of $G L(F, A)$ this is no longer the case. The corresponding counterexamples were constructed in [13]. Therefore the following questions arise naturally.

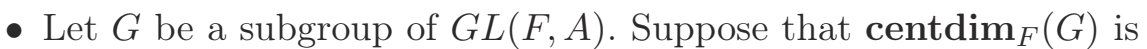
finite. For which groups $G$ does it follow that $\operatorname{augdim}_{F}(G)$ is finite?

- Let $G$ be a subgroup of $G L(F, A)$. Suppose that $\operatorname{augdim}_{F}(G)$ is finite. For which groups $G$ does it follow that $\operatorname{centdim}_{F}(G)$ is finite?

The answers to these questions have been obtained in the paper [13].

In the examples constructed in [13], the group $G$ was either an infinite elementary abelian $p$-group or a free abelian group with infinite $\mathbb{Z}$-rank. In the latter case the factor group $G / G^{p}$ is an infinite elementary abelian $p$-group for each prime $p$. One might hope that in the absence of such infinite sections positive answers to the two questions might then be obtained and this is indeed the case.

Let $p$ be a prime. We say that a group $G$ has finite section $p$-rank $\mathbf{s r}_{p}(G)=r$ if every elementary abelian $p$-section of $G$ is finite of order at most $p^{r}$ and there is an elementary abelian $p$-section $A / B$ of $G$ such that $|A / B|=p^{r}$.

Groups of finite section $p$-rank have been extensively studied and detailed information concerning such groups can be found in the book [17]. The section $p$-rank is closely linked to the special rank mentioned earlier.

The main results of [13] are as follows.

Theorem 6. Let $A$ be a vector space over the field $F$, of characteristic the prime $p$, and let $G$ be a subgroup of $G L(F, A)$. Suppose that $\operatorname{centdim}_{F}(G)=\operatorname{codim}_{F}\left(\zeta_{G}(A)\right)=c$ is finite. If $G$ has finite section p-rank $r$, then $\operatorname{augdim}_{F}(G)=\operatorname{dim}_{F}([G, A])$ is finite. Furthermore, there 
exists a function $\kappa_{1}$ such that

$$
\operatorname{augdim}_{F}(G)=\operatorname{dim}_{F}([G, A]) \leqslant \kappa_{1}(c, r) .
$$

Theorem 7. Let $A$ be a vector space over a field $F$ of characteristic 0 and let $G$ be a subgroup of $G L(F, A)$. Suppose that centdim $_{F}(G)=$ $\operatorname{codim}_{F}\left(\zeta_{G}(A)\right)=d$ is finite. If $G$ has finite section $p$-rank $r$ for some prime $p$, then $\operatorname{augdim}_{F}(G)=\operatorname{dim}_{F}([G, A])$ is finite. Furthermore, there exists a function $\kappa_{2}$ such that

$$
\operatorname{dim}_{F}([G, A]) \leqslant \kappa_{2}(d, r) .
$$

These theorems can be regarded as analogues for linear groups of the well-known group theoretical theorem known as Schur's Theorem (although Schur did not prove this result-see the paper [39]). This theorem first appeared in the work of B. H. Neumann [44] and can be stated as follows.

- If the center of a group $G$ has finite index, then the derived subgroup of $G$ is finite.

The converse of this result is false since there are infinite groups with finite center and finite derived subgroup. However P. Hall [19] proved

- If the derived subgroup of a group $G$ is finite, then the second center of $G$ has finite index.

In connection with this result and the above results, the following question arises naturally:

- Let $G$ be a subgroup of $G L(F, A)$. Suppose that $\operatorname{dim}_{F}([G, A])=c$ is finite. For which groups $G$ does it follow that $\operatorname{dim}_{F}\left(A / \zeta_{G}(A)\right)$ is finite?

The finiteness condition of section $p$-rank is quite strong. In the presence of this finiteness condition it is possible to obtain a stronger result for linear groups than just an analogue of Hall's theorem quoted above. This result was obtained in [15] and is as follows.

Theorem 8. Let $A$ be a vector space over a field $F$ of prime characteristic $p$ and let $G$ be a subgroup of $G L(F, A)$. Suppose that $\operatorname{augdim}_{F}(G)=c$ is finite. If $G$ has finite section p-rank $r$, then centdim $_{F}(G)$ is finite. Furthermore,

$$
\operatorname{centdim}_{F}(G) \leqslant \kappa_{1}(c, r) .
$$

Theorem 9. Let $A$ be a vector space over a field $F$ of characteristic 0 and let $G$ be a subgroup of $G L(F, A)$. Suppose that augdim $_{F}(G)=c$ is finite. 
If $G$ has finite section $p$-rank $r$ for some prime $p$, then centdim ${ }_{F}(G)$ is finite. Furthermore,

$$
\operatorname{centdim}_{F}(G) \leqslant \kappa_{2}(c, r) .
$$

The theorem concerning groups whose centers have finite index, mentioned above, has significant generalizations. The first of these generalizations was obtained in the work of $\mathrm{R}$. Baer [1]. A natural next step in the context of linear groups is to obtain an analogue of this result of Baer's, using the same restrictions that were used to prove the analogue of the theorem of B. H. Neumann. First it is appropriate to recall the following concepts.

Starting from the $G$-center we can construct the upper $G$-central series

$$
0=\zeta_{G, 0}(A) \leqslant \zeta_{G, 1}(A) \leqslant \ldots \zeta_{G, \alpha}(A) \leqslant \zeta_{G, \alpha+1}(A) \leqslant \ldots \zeta_{G, \gamma}(A)
$$

of $A$, where $\zeta_{G, 1}(A)=\zeta_{G}(A), \zeta_{G, \alpha+1}(A) / \zeta_{G, \alpha}(A)=\zeta_{G}\left(A / \zeta_{G, \alpha}(A)\right)$, for all ordinals $\alpha, \zeta_{G, \lambda}(A)=\bigcup_{\beta<\lambda} \zeta_{G, \beta}(A)$, for all limit ordinals $\lambda$ and $\zeta_{G}\left(A / \zeta_{G, \gamma}(A)\right)=0$

From this definition we see that $\left[G, \zeta_{G, \alpha+1}(A)\right] \leqslant \zeta_{G, \alpha}(A)$ for each ordinal $\alpha<\gamma$.

The last term $\zeta_{G, \gamma}(A)$ of the series is called the upper $G$-hypercenter of $A$ and is denoted by $\zeta_{G, \infty}(A)$.

The ordinal $\gamma$ is called the $G$-central length of $A$ and is denoted by $\mathbf{z l}_{G}(A)$.

An ascending series of $G$-invariant subspaces

$$
0=A_{0} \leqslant A_{1} \leqslant \ldots A_{\alpha} \leqslant A_{\alpha+1} \leqslant \ldots A_{\gamma}
$$

is said to be $G$-central if $\left[G, A_{\alpha+1}\right] \leqslant A_{\alpha}$ for each ordinal $\alpha<\gamma$. It is easy to see that if $A$ has a $G$-central series of length $\gamma$, then $\mathbf{z l}_{G}(A) \leqslant \gamma$.

We say that a vector space $A$ is $G$-hypercentral if $A=\zeta_{G, \infty}(A)$ and we then say that $G$ acts hypercentrally on $A$.

We note that $A$ is $G$-hypercentral if and only if $A$ has an ascending series of $G$-invariant subspaces, the last term of which is $A$, and for which $G$ acts trivially on each of the factors.

When $A$ is a vector space over a field $F$ and $G$ is a subgroup of $G L(F, A)$ we define the lower $G$-central series of $A$,

$$
A=\gamma_{G, 1}(A) \geqslant \gamma_{G, 2}(A) \geqslant \ldots \gamma_{G, \alpha}(A) \geqslant \gamma_{G, \alpha+1}(A) \geqslant \ldots \gamma_{G, \kappa}(A),
$$


by

$$
\begin{aligned}
\gamma_{G, 2}(A) & =[G, A]=\left[G, \gamma_{G, 1}(A)\right], \\
\gamma_{G, \alpha+1}(A) & =\left[G, \gamma_{G, \alpha}(A)\right], \text { for all ordinals } \alpha \\
\gamma_{G, \lambda}(A) & =\bigcap_{\beta<\lambda} \gamma_{G, \beta}(A) \text { for all limit ordinals } \lambda
\end{aligned}
$$

and

$$
\gamma_{G, \kappa}(A)=\left[G, \gamma_{G, \kappa}(A)\right] .
$$

It clearly follows from the definition that

$$
\gamma_{G, \alpha}(A) / \gamma_{G, \alpha+1}(A) \leqslant \zeta_{G}\left(A / \gamma_{G, \alpha+1}(A)\right) \text { for each ordinal } \alpha<\kappa \text {. }
$$

The last term $\gamma_{G, \kappa}(A)$ of this series is called the lower $G$-hypocenter of $A$ and is denoted by $\gamma_{G, \infty}(A)$.

We say that the $A$ is $G$-hypocentral if $\gamma_{G, \infty}(A)=0$.

Theorem 10. Let $A$ be a vector space over a field $F$ of prime characteristic $p$ and let $G$ be a subgroup of $G L(F, A)$. Suppose that there is a natural number $n$ such that $\operatorname{codim}_{F}\left(\zeta_{G, n}(A)\right)=c$ is finite. If $G$ has finite section p-rank $r$, then $\operatorname{dim}_{F}\left(\gamma_{G, n+1}(A)\right)$ is finite and there is a function $\kappa_{3}$ such that $\operatorname{dim}_{F}\left(\gamma_{G, n+1}(A)\right) \leqslant \kappa_{3}(c, r, n)$.

Theorem 11. Let $A$ be a vector space over a field $F$ of characteristic 0 and let $G$ be a subgroup of $G L(F, A)$. Suppose that there is a natural number $n$ such that $\operatorname{codim}_{F}\left(\zeta_{G, n}(A)\right)=d$ is finite. If $G$ has finite section p-rank $r$ for some prime $p$, then $\operatorname{dim}_{F}\left(\gamma_{G, n+1}(A)\right)$ is finite and there is a function $\kappa_{4}$ such that $\operatorname{dim}_{F}\left(\gamma_{G, n+1}(A)\right) \leqslant \kappa_{4}(d, r, n)$

These results appeared in the paper [13]. We immediately note the dual results which have been obtained in [15].

Theorem 12. Let $A$ be a vector space over a field $F$ of prime characteristic $p$ and let $G$ be a subgroup of $G L(F, A)$. Suppose that there is a natural number $n$ such that $\operatorname{dim}_{F}\left(\gamma_{G, n+1}(A)\right)=c$ is finite. If $G$ has finite section $p$ rank $r$, then $\operatorname{codim}_{F}\left(\zeta_{G, n}(A)\right)$ is finite and $\operatorname{codim}_{F}\left(\zeta_{G, n}(A)\right) \leqslant \kappa_{3}(c, r, n)$.

Theorem 13. Let $A$ be a vector space over a field $F$ of characteristic 0 and let $G$ be a subgroup of $G L(F, A)$. Suppose that there is a natural number $n$ such that $\operatorname{dim}_{F}\left(\gamma_{G, n+1}(A)\right)=d$ is finite. If $G$ has finite section p-rank $r$, then $\operatorname{codim}_{F}\left(\zeta_{G, n}(A)\right)$ is finite. Furthermore,

$$
\operatorname{codim}_{F}\left(\zeta_{G, n}(A)\right) \leqslant \kappa_{4}(d, r, n) .
$$


A generalization of the result of P. Hall was obtained in [18] where it was proved that a group $G$ has a finite normal subgroup $K$ such that $G / K$ is hypercentral if and only if the upper hypercenter of $G$ has finite index. Linear analogues of this result also hold and we now give these results which were obtained in [14].

We first need some terminology: When $A$ is a vector space over a field $F$ and $G$ is a subgroup of $G L(F, A)$, we say that $A$ is $G$-locally nilpotent if for every finitely generated subgroup $H$ of $G$ and every finite subset $M$ of $A$ the $F H$-submodule generated by $M,(F H) M$, is $H$-nilpotent. Let $\mathcal{L}$ denote the family of $G$-invariant subspaces $X$ of $A$ such that $A / X$ is $G$-locally nilpotent. The intersection of all members of the family $\mathcal{L}$ is called the G-locally nilpotent residual of $A$.

Theorem 14. Let $A$ be a vector space over a field $F$ of prime characteristic p. Let $G$ be a subgroup of $G L(F, A)$. If $\operatorname{codim}_{F}\left(\zeta_{G, \infty}(A)\right)=d$ is finite and if $G$ has finite section $p$-rank $r$, then the $G$-locally nilpotent residual $L$ of $A$ has finite dimension at most

$$
\frac{1}{2}\left(5 d^{3}+d^{2}+6 d+6 r d\right) .
$$

Furthermore, $A / L$ is $G$-hypercentral.

Theorem 15. Let $A$ be a vector space over a field $F$ of characteristic 0 and let $G$ be a subgroup of $G L(F, A)$. Suppose that

$$
\operatorname{codim}_{F}\left(\zeta_{G, \infty}(A)\right)=d
$$

is finite. If $G$ has finite section $p$-rank $r$, for some prime $p$, then the $G$-locally nilpotent residual $L$ of $A$ has finite dimension at most $3 d+3 r d$. Furthermore, $A / L$ is G-hypercentral.

We also have the following dual theorems which appeared in [15]:

Theorem 16. Let $A$ be a vector space over a field $F$ of prime characteristic $p$ and let $G$ be a subgroup of $G L(F, A)$. Suppose that $A$ contains a $G$ invariant subspace $B$ such that $\operatorname{dim}_{F}(B)=d$ is finite and that $A / B$ is $G$ hypercentral. If $G$ has finite section p-rank $r$, then the upper $G$-hypercenter $Z$ of $A$ has finite codimension at most

$$
\frac{1}{2}\left(5 d^{3}+d^{2}+6 d+6 r d\right)
$$


Theorem 17. Let $A$ be a vector space over a field $F$ of characteristic 0 and let $G$ be a subgroup of $G L(F, A)$. Suppose that $A$ contains a $G$-invariant subspace $B$ such that $\operatorname{dim}_{F}(B)=d$ is finite and $A / B$ is $G$-hypercentral. If $G$ has finite section p-rank $r$, for some prime $p$, then the upper $G$ hypercenter $Z$ of $A$ has finite codimension at most $3 d+3 r d$.

\section{Groups with finite orbits}

The structure of a linear group $G$ is significantly affected by its family of $G$-invariant subspaces, the properties of its elements, the sizes, location and saturation of the family of all subspaces, $G$-invariant subspaces and so on. For example, consider the following extreme situation.

Let $F$ be a field, $A$ a vector space over $F$ and let $G$ be a subgroup of $G L(F, A)$. Suppose that every subspace of $A$ is $G$-invariant. In particular, for each element $a$ of $A$ the subspace $F a$ is $G$-invariant. If $f, g$ are arbitrary elements of $G$, then $f(a)=\alpha a, g(a)=\beta a$, for some $\alpha, \beta \in F$. Then

$$
(f g)(a)=f(g(a))=f(\beta a)=\beta f(a)=\beta \alpha a ; \quad \text { likewise }(g f)(a)=\alpha \beta a .
$$

Since the multiplicative group of $U(F)$ of $F$ is abelian it follows that $f g=g f$ so that in this case $G$ must be abelian.

This simple example nevertheless gives a good illustration of the influence of a family of $G$-invariant subspaces on the structure of a linear group $G$. Figuratively speaking, if the family of $G$-invariant subspaces is quite large, then the group $G$ is to a greater or lesser degree close to abelian. For a linear group $G$ we define the following $G$-invariant subspace.

$\mathbf{F O}_{G}(A)=\left\{a \in A \mid a^{G}\right.$ is finite $\}=\left\{a \in A|| G: C_{G}(a) \mid\right.$ is finite $\}$.

Next we consider the situation when $A=\mathbf{F O}_{G}(A)$.

We shall say that the linear group $G$ has finite orbits on $A$ if the orbits $a^{G}$ are finite for every element $a \in A$. If the $G$-orbit of $a$ is finite, then $\left|a^{G}\right|=\left|G: C_{G}(a)\right|$.

For the group $G$, one of the natural actions of $G$ on itself is the action of conjugation by a given element. Furthermore, the orbit of $g \in G$ is the conjugacy class $\left\{x^{-1} g x=g^{x} \mid x \in G\right\}$. Hence we may consider the class of linear groups having finite orbits on $A$ as an analogue of the class of FC-groups, which has been studied extensively. We note that

1) If $G$ is a subgroup of $G L(F, A)$ having finite orbits on $A$, then $G$ is residually finite. Conversely, if $G$ is a residually finite group, then 
for each field $F$ there exists a vector space $V$ over $F$ such that $G$ has finite orbits on $V$.

Thus the fact that the orbits of all elements are finite is equivalent to the finite approximability (residual finiteness) of the group. The theory of residually finite groups is quite well established and has many interesting results, but this theory is not really near completion. Returning to our analogy between linear groups having finite orbits and FC-groups, the same can also be said concerning the theory of FC-groups. In the theory of FC-groups some subclasses of groups emerged naturally and occupied a special place in this theory. One such subclass appeared in the paper [45], for example. In this paper B. H. Neumann discussed the class of groups in which the conjugacy classes are finite but of bounded size. Neumann called such groups BFC-groups and proved that such groups are finite-by-abelian. Thus in this case the derived subgroup $G^{\prime}$ of the BFC-group $G$ is finite. There is a natural analogue of such groups when we discuss linear groups.

Let $A$ be a vector space over the field $F$ and let $G$ denote a subgroup of $G L(F, A)$. We say that $G$ has boundedly finite orbits on $A$ if there is a natural number $b$ such that $\left|a^{G}\right| \leqslant b$, for each element $a$ of $A$.

We let $\mathbf{I O}_{A}(G)=\max \left\{\left|a^{G}\right| \mid a \in A\right\}$ and first discuss some simple situations when the values of $\mathbf{I O}_{A}(G)$ are small.

If $\mathbf{I O}_{A}(G)=1$, then $a^{G}=\{a\}$ for each element $a \in A$. It follows that $g a=a$ for all $g \in G$. Thus in this case $G$ is the trivial group.

Suppose that $\mathbf{I O}_{A}(G)=2$ and let $a$ be an element of $A$ such that $a^{G} \neq\{a\}$. In this case the group $G$ contains an element $g$ such that $g a \neq a$. For the element $g^{2} a=g(g(a))$ we have two choices, namely $g^{2} a=a$ or $g^{2} a=g a$. However, in the latter case we immediately deduce that $g a=a$, contradicting the choice of $g$. Thus $g^{2} a=a$. If $d$ is a further element of $A$ such that $g d \neq d$, then the above argument can be repeated to deduce that $g^{2} d=d$. Consequently, for each arbitrary element $c$ of the space $A$ we have $g c=c$ or $g^{2} c=c$. It follows that $g$ has order 2, so that in this case the group $G$ is an elementary abelian 2-group.

Taking into account the result of B. H. Neumann, mentioned above, concerning the structure of BFC-groups and the natural similarity between the derived subgroup and the $G$-commutator subspace of a vector space we pose the following very natural question.

- Let $F$ be a field, let $A$ be a vector space over $F$ and let $G$ be a subgroup of $G L(F, A)$. Suppose that $G$ has boundedly finite orbits on $A$. Is it then the case that the $G$-commutator subspace $[G, A]$ has finite dimension over $F$ ? 
We note at once however that the answer to this question is negative. The corresponding counterexamples were obtained in [11]. This appears to show that the situation of linear groups having boundedly finite orbits looks more complicated than the situation for BFC-groups.

We now give the results that were obtained in [11]. In that paper we considered the more general situation of modules over group rings, but here we restrict ourselves to considering only the case of linear groups.

The main structural details of linear groups having boundedly finite orbits are exhibited in the following result.

Theorem 18. Let $A$ be a vector space over a field $F$ and let $G$ be a subgroup of $G L(F, A)$ having boundedly finite orbits on $A$. Suppose that $I \boldsymbol{O}_{A}(G)=b$. Then $G$ contains a normal subgroup $L$ and $A$ contains a $G$-invariant subspace $C$ satisfying the following conditions:

(i) L has finite index in $G$;

(ii) $C$ has finite dimension;

(iii) $[L, A] \leqslant C$ and $[L, C]=0$;

(iv) if $\operatorname{char}(F)=p$, a prime, then $L$ is an elementary abelian p-group; if $\operatorname{char}(F)=0$, then $L=1$ and $G$ is finite.

Moreover, there are functions $\beta_{3}$ and $\beta_{4}$ such that $|G: L| \leqslant \beta_{3}(b)$ and $\operatorname{dim}_{F}(C) \leqslant \beta_{4}(b)$.

Here are some further details concerning the structure.

Theorem 19. Let $A$ be a vector space over a field $F$ and let $G$ be a subgroup of $G L(F, A)$ having boundedly finite orbits on $A$. Suppose that $I_{O_{A}}(G)=b$. Suppose also that $G$ is a periodic $p^{\prime}$-subgroup whenever $F$ has prime characteristic $p$. Then $G$ is finite of order at most $(b !)^{b}$.

Corollary 4. Let $A$ be a vector space over a field $F$ of characteristic 0 and let $G$ be a subgroup of $G L(F, A)$ having boundedly finite orbits on $A$. Suppose that $\boldsymbol{I O}_{A}(G)=b$. Then $G$ is finite and $|G| \leqslant(b !)^{b}$.

Corollary 5. Let $A$ be a vector space over a field $F$ of prime characteristic $p$ and let $G$ be a subgroup of $G L(F, A)$ having boundedly finite orbits on A. Suppose that $\boldsymbol{I O}_{A}(G)=b$. Then every $p^{\prime}$-subgroup of $G$ is finite of order at most $(b !)^{b}$.

As we saw above, the condition that the $G$-orbits of the linear group $G$ are finite is a rather strong one. If the orbit $a^{G}$ of an element $a \in A$ is finite, then the dimension of the subspace of $A$ generated by $a^{G}$ is also finite. However it is clear that the converse of this is not true, because there are finite dimensional vector spaces having infinite $G$-orbits. 
For the linear group $G$ we define a $G$-invariant subspace

$$
\mathbf{F D O}_{G}(A)=\left\{a \in A \mid F a^{G} \text { is finite dimensional }\right\} .
$$

We now consider the situation in which $A=\mathbf{F D O}_{G}(A)$, that is the case in which all subspaces $F a^{G}$ are finite dimensional for all $a \in A$.

We say that a linear group has finite dimensional orbits (on $A$ ) if the $G$-orbit $a^{G}$ of every element $a \in A$ generates a finite dimensional subspace.

Here we also have an analogy with the class of FC-groups, although now the analogy is much weaker.

As before, the fact that the orbits of all the elements of a vector space are finite dimensional is equivalent to saying that a linear group acting in this way is residually (finite dimensional). The class of such groups is very much broader than the class of residually finite groups.

If we turn again to the analogy with FC-groups, we can see that another natural analogue of BFC-groups will be that of linear groups $G$ acting on the vector space $A$ in such a way that each $G$-orbit generates a finite dimensional subspace having dimension bounded by some natural number $b$. Such linear groups were discussed in the paper [12]. We shall present the main results of this paper here. Naturally the situation here is more complicated and, unlike with boundedly finite orbits, the case of fields of characteristic 0 plays a more interesting role.

As usual, let $F$ be a field, let $A$ be a vector space over the field $F$ and let $G$ be a subgroup of $G L(F, A)$. We shall say that $G$ has boundedly finite dimensional orbits on $A$ if there is a natural number $b$ such that $\operatorname{dim}_{F}\left(F a^{G}\right) \leqslant b$ for each element $a$ of the vector space $A$.

In this case we let $\operatorname{do}_{A}(G)=\max \left\{\operatorname{dim}_{F}\left(F a^{G}\right) \mid a \in A\right\}$.

We first consider the case when $\operatorname{do}_{A}(G)=1$, so that in this case each of the subspaces $F a^{G}$ is one dimensional. Naturally, in this case, $F a$ is a subspace of $F a^{G}$ and it follows that $F a^{G}=F a$. Thus in this case every subspace $F a$ is $G$-invariant.

Theorem 20. Let $A$ be a vector space over a field $F$ and let $G$ be a subgroup of $G L(F, A)$ having boundedly finite dimensional orbits on A. Suppose also that $\boldsymbol{d o}_{A}(G)=b>1$. Then

(i) A contains a $G$-invariant subspace $D$ of finite dimension such that every subspace of $A / D$ is $K$-invariant, where $K=C_{G}(D)$. Moreover, there is a function $\beta_{5}$ such that $\operatorname{dim}_{F}(D) \leqslant \beta_{5}(b)$;

(ii) $K$ contains a $G$-invariant subgroup $L$ such that $[L, A] \leqslant D$ and $K / L$ is isomorphic to a subgroup of $U(F)$; 
(iii) if $\operatorname{char}(F)=p$, a prime, then $L$ is an elementary abelian $p$-group and if $\operatorname{char}(F)=0$, then $L$ is an abelian torsion-free subgroup.

Corollary 6. Let $A$ be a vector space over a field $F$ and let $G$ be a periodic subgroup of $G L(F, A)$ having boundedly finite dimensional orbits on $A$. Then $G$ is locally finite.

Corollary 7. Let $A$ be a vector space over a field $F$ and let $G$ be a subgroup of $G L(F, A)$ having boundedly finite dimensional orbits on A. If $G$ contains no non-cyclic free subgroups then $G$ is soluble-by-locally finite. Furthermore, if $\operatorname{char}(F)=0$, then $G$ is soluble-by-finite.

Corollary 8. Let $A$ be a vector space over a field $F$ and let $G$ be a periodic subgroup of $G L(F, A)$ having boundedly finite dimensional orbits on $A$. Suppose that if $\operatorname{char}(F)=p$, a prime, then $G$ is a $p^{\prime}$-group. Then the center of $G$ contains a locally cyclic subgroup $K$ such that $G / K$ is abelianby-finite.

Let $A$ be a vector space over a field $F$, let $B$ be a subspace of $A$ and let $G$ be a subgroup $G L(F, A)$. For every element $g \in G$, we let

$$
g B=g(B)=\{g b \mid b \in B\} .
$$

Clearly $g B$ is a subspace of $A$. Just as we defined the $G$-orbits for the elements of $A$ we can do the same for subspaces.

Indeed, we define the $G$-orbits of a subspace $B$ of $A$ to be the family $B^{G}=\{g B \mid g \in G\}$.

If $V$ is a subspace of $A$, then we define the invariator of $V$ in $G$ by

$$
\operatorname{Inv}_{G}(V)=\{g \in G \mid g(v) \in V \text { for every element } v \in V\} .
$$

It is easy to see that $\operatorname{Inv}_{G}(V)$ is a subgroup of $G$ and that $V$ is $G$-invariant if and only if $\operatorname{Inv}_{G}(V)=G$. Clearly also the intersection of an arbitrary family of $G$-invariant subspaces is a $G$-invariant subspace.

If the $G$-orbit of a subspace $B$ is finite, then we can show that $\left|B^{G}\right|=$ $\left|G: \operatorname{Inv}_{G}(B)\right|$.

Returning again to the analogy with FC-groups, another classic result of B. H. Neumann immediately comes to mind. In the paper [46] Neumann proved that if the subgroups of an abstract group $G$ have a finite number of conjugates (so have finite $G$-orbits under conjugation) then the group is central-by-finite. Thus we arrive at the problem of describing the structure of linear groups having finite $G$-orbits of subspaces. 
Suppose that the $G$-orbit of every subspace $B$ of a vector space $A$ is finite. Then the $G$-orbit of every one dimensional subspace $F a$ is finite for each $a \in A$. From what we noted above it then follows that $\mid G$ : $\operatorname{Inv}_{G}(F a) \mid$ is finite. Let $T$ be a transversal to $\operatorname{Inv}_{G}(F a)$ in the group $G$. Then $G(F a)=F a^{G}=\sum_{g \in T} g(F a)$ and the finiteness of $T$ implies that the $G$-invariant subspace $G(F a)$ has finite dimension. Furthermore $\operatorname{dim}_{F}(G(F a)) \leqslant|T|$. If we now suppose that there is a natural number $b$ such that $\left|B^{G}\right| \leqslant b$ for each subspace $B$, then we deduce that the group $G$ has boundedly finite dimensional orbits of elements and we arrive at the situation that has already been discussed previously. Consequently, we shall consider the situation when the orbits of subspaces are finite without assuming, however, that the sizes of these orbits are bounded. On the other hand, the theorem of B. H. Neumann mentioned above implies that if the sizes of the conjugacy classes of subgroups of a group are finite, then their sizes are bounded. This prompts the following very natural question:

- Let $A$ be a vector space over a field $F$ and let $G$ be a subgroup of $G L(F, A)$. Suppose that the $G$-orbit of every subspace $B$ of $A$ is finite. In this case, will the sizes of these orbits be bounded?

The structure of linear groups having finite orbits of subspaces was obtained in [12] as follows:

Theorem 21. Let $A$ be a vector space over a field $F$ and let $G$ be a subgroup of $G L(F, A)$. Suppose that the $G$-orbit of every subspace of $A$ is finite. Then the following conditions hold:

(i) $G$ contains a normal subgroup $K$ of finite index such that every subspace of $A$ is $K$-invariant;

(ii) $G$ is center-by-finite;

(iii) there is a natural number $k$ such that the $G$-orbit of every subspace of $A$ contains at most $k$ subspaces.

In particular $G$ has boundedly finite orbits of subspaces.

\section{Maximal and minimal conditions on subgroups of infinite central dimension}

As we saw earlier a definitive study of infinite dimensional linear groups is really presently only possible in the presence of certain restrictions. The question arises as to what type of restrictions will be useful here. This situation is similar to the one that occurred during the time when the theory of infinite groups was in its infancy. At that time the theory of finite groups was already quite well established and many of its fundamental 
results had been obtained. It was therefore natural to use the experience already accumulated in the theory of finite groups when trying to study infinite groups. Thus, for example, in the theory of infinite groups a large amount of work has been done concerning groups with finiteness conditions where many of the results of finite group theory have been used to generalize that theory. In this way group theory has been enriched with many new fundamental results and techniques.

The theory of finite dimensional linear groups is one of the most developed algebraic theories, so it is natural to rely on this theory when building the theory of infinite dimensional linear groups and in this way we come to an approach based on finite dimensional conditions. The effectiveness of this approach has already been demonstrated in the theory of finitary linear groups (see the survey paper of R. E. Phillips [50], for example). We now demonstrate the effectiveness of this approach as applied to other types of infnite dimensonal linear groups.

One of the "largest" families of a group $G$ is the family of all proper subgroups of $G$. The study of groups whose proper subgroups have some fixed property $\mathcal{P}$ began with the classical work of R. Dedekind [6]. In this work he considered those finite groups whose proper subgroups are normal. Shortly afterwards, in their famous paper [42], G. Miller and H. Moreno described those finite groups all of whose proper subgroups are abelian. In this setting, we need to mention the remarkable article [51] of O. Yu. Schmidt, which completely describes those finite groups all of whose proper subgroups are nilpotent. In order to develop the theory of infinite groups further, the following problem of O. Yu. Schmidt was very significant:

- Describe those infinite groups whose proper subgroups are finite.

This problem was formulated during the 1930's. This was the time when the theory of infinite groups began to develop intensively at Moscow University. Schmidt 's problem turned out to be the starting point of a whole new branch of the theory of infinite groups, the essence of which was the study of groups with different minimal conditions.

A whole series of articles was devoted to solving the Schmidt problem. Early "positive" results were obtained by M. I. Kargapolov [22] and P. Hall and C. R. Kulatilaka [20]. They showed that an infinite locally finite group whose proper subgroups are finite is a Prüfer $p$-group for some prime $p$. In general the situation was rather complicated In the article [48], A. Yu. Olshanskii constructed an infinite periodic group whose proper subgroups all have prime order and in [49], A. Yu. Olshanskii constructed 
an infinite $p$-group, where $p$ is a prime greater than $10^{75}$, all of whose proper subgroups have order $p$.

Based on such considerations the following natural problem can therefore be posed for infinite dimensional linear groups:

- Describe the infinite dimensional linear groups whose proper subgroups have finite central dimension.

This problem was considered in the paper [7] where a somewhat more general situation was considered.

Let $G$ be a subgroup of $G L(F, A)$ of infinite central dimension and let $\mathcal{L}_{i c d}(G)$ denote the family of subgroups of $G$ having infinite central dimension. We say that $G$ satisfies the minimal condition on subgroups of infinite central dimension, which we shorten to min-icd, if the family $\mathcal{L}_{i c d}(G)$, ordered by inclusion, satisfies the minimal condition.

In particular, if every proper subgroup of $G$ has finite central dimension, then $\mathcal{L}_{i c d}(G)=\{G\}$.

The paper [7] was devoted to the study of infinite dimensional linear groups satisfying the condition min-icd and here we present the results of this work.

Theorem 22. Let $A$ be a vector space over the field $F$ and let $G$ be a subgroup of $G L(F, A)$ which has infinite central dimension. If $G$ satisfies the condition min-icd, then either $G$ has the minimal condition on all subgroups or $G$ is a finitary linear group.

Theorem 23. Let $A$ be a vector space over the field $F$ of prime characteristic $p$ and let $G$ be a (locally soluble)-by-finite subgroup of $G L(F, A)$ having infinite central dimension. Suppose that $G$ satisfies the condition min-icd and that $G$ is not Chernikov. Then $G$ contains normal subgroups $P \leqslant D \leqslant G$ satisfying the following conditions:

(i) $P$ is a bounded nilpotent p-group of finite central dimension;

(ii) $D=P \rtimes Q$ for some non-trivial divisible Chernikov $p^{\prime}$-subgroup $Q$;

(iii) $Q$ has infinite central dimension;

(iv) $P$ satisfies the minimal condition on $Q$-invariant subgroups;

(v) $G / D$ is finite.

In particular $G$ is a nilpotent-by-abelian-by-finite group and satisfies the minimal condition on normal subgroups.

Corollary 9. Let $A$ be a vector space over the field $F$ of prime characteristic $p$ and let $G$ be a (locally soluble)-by-finite subgroup of $G L(F, A)$ having infinite central dimension. Suppose that every proper subgroup of $G$ has finite central dimension. Then $G$ is a Prüfer q-group for some prime $q$. 
Theorem 24. Let $A$ be a vector space over the field $F$ of characteristic 0 and let $G$ be a (locally soluble)-by-finite subgroup of $G L(F, A)$ having infinite central dimension. Suppose that $G$ satisfies the condition min-icd. Then $G$ is a Chernikov group.

Theorem 25. Let $A$ be a vector space over the field $F$ and let $G$ be a locally finite subgroup of $G L(F, A)$ having infinite central dimension. If $G$ satisfies the condition min-icd, then $G$ is a soluble-by-finite group.

Theorem 26. Let $A$ be a vector space over the field $F$ and let $G$ be a locally generalized radical subgroup of $G L(F, A)$ having infinite central dimension. If $G$ satisfies the condition min-icd, then $G$ is a soluble-by-finite group.

The maximal condition on ordered sets is dual to the minimal condition. Above we saw how to handle the condition min-icd using the analogy with the problem of S. N. Chernikov concerning groups with the minimal condition for all subgroups. As we saw a significant portion of the groups in the class of linear groups satisfying min-icd also satisfy the minimal condition for all subgroups. For groups satisfying the maximal condition on all subgroups R. Baer also formulated the problem of whether such groups would be polycyclic-by-finite. The maximal condition is somewhat more intractible than the minimal condition. Thus it is fair to say that the problem of S. N. Chernikov received more attention. A negative solution to the Baer problem was also obtained in the work of A. Yu. Olshanskii in [47]. Nevertheless, the maximal condition has played a very important role in the development of the theory of infinite groups. The study of groups with the maximal condition on various types of subgroup enriched the theory of infinite groups with ideas, techniques, constructions and various interesting and deep results.

Let $G$ be a subgroup of $G L(F, A)$. We say that $G$ satisfies the maximal condition on subgroups of infinite central dimension, which we shorten to max-icd, if the family $\mathcal{L}_{i c d}(G)$, ordered by inclusion, satisfies the maximal condition.

Linear groups satisfying the condition max-icd were studied in the paper [38] and it is to this that we now turn attention. As we shall see, the situation here is much more diverse (as is often typically the case for the maximal conditions).

When studying linear groups satisfying the maximal condition on subgroups of infinite central dimension, two cases naturally arise, namely when the corresponding group has no finite generating set and when it is 
finitely generated. The consideration of the first situation consists of two stages.

Theorem 27. Let $A$ be a vector space over the field $F$ and let $G$ be a soluble subgroup of $G L(F, A)$ satisfying max-icd. Suppose that $G$ has infinite central dimension and that the factor group $G / G^{\prime}$ is not finitely generated. Then $G$ contains normal subgroups $K, V$ such that $K \leqslant V$ satisfying the following conditions:

(i) $G / V$ is a Prüfer q-group for some prime $q$, the factor group $G / K$ is abelian-by-finite and $V / K$ is finitely generated. Furthermore $K$ is nilpotent and the vector space $A$ is $K$-nilpotent;

(ii) if $\operatorname{char}(F)=p$ a prime, then $q \neq p$ and $K$ is a bounded $p$-subgroup;

(iii) if $\operatorname{char}(F)=0$, then $K$ is torsion-free;

(iv) there exists a field extension $E$ of the field $F$ such that the multiplicative group of the field $E$ contains a Prüfer $q$-subgroup;

(v) the subspace $C=C_{A}(V)$ has finite codimension in $A$;

(vi) $C$ contains a $G$-invariant subspace $B$ having a direct decomposition $B=\bigoplus_{n \in \mathbb{N}} B_{n}$, where $B_{n}$ is a $G$-invariant subspace of $A$ having finite dimension for each natural number $n$. Furthermore, $[G, C] \leqslant B$ and $\operatorname{dim}_{E}\left(B_{n}\right)=1$ for each $n \in \mathbb{N}$.

Theorem 28. Let $A$ be a vector space over the field $F$ and let $G$ be a soluble subgroup of $G L(F, A)$ satisfying max-icd. Suppose that $G$ has infinite central dimension and that $G$ is not finitely generated. Then $G$ contains normal subgroups $K \leqslant W \leqslant G$ satisfying the following conditions:

(i) $G / W$ is finitely generated and abelian-by-finite;

(ii) $W / K$ is abelian;

(iii) $W$ contains a subgroup $L$ such that $L / K$ is finitely generated and $W / L$ is a Prüfer q-group for some prime $q$;

(iv) $K$ is nilpotent and the vector space $A$ is $K$-nilpotent.

The next natural problem to study is that of finitely generated groups satisfying the maximal condition on subgroups having infinite central dimension. Let $G$ be a subgroup of $G L(F, A)$ and let $\operatorname{Fin}(G)$ denote the subset of $G$ consisting of all elements $g$ of the group $G$ for which the cyclic subgroup $\langle g\rangle$ has finite central dimension. The normal $\operatorname{subgroup} \operatorname{Fin}(G)$ is called the finitary radical of the linear group $G$. Here we also have two cases.

Theorem 29. Let $A$ be a vector space over the field $F$ and let $G$ be a finitely generated soluble subgroup of $G L(F, A)$ satisfying max-icd. Sup- 
pose that $G$ has infinite central dimension but that its finitary radical has finite central dimension. Then the following conditions hold:

(i) $G$ contains a normal subgroup $U$ such that $G / U$ is polycyclic;

(ii) there is a natural number $m$ such that $(x-1)^{m}=0$ for each element $x \in U$, so that every element of $U$ is unipotent and the subgroup $U$ is nilpotent;

(iii) if $\operatorname{char}(F)=0$, then $U$ is torsion-free; if $\operatorname{char}(F)=p$, a prime, then $U$ is bounded;

(iv) $U$ satisfies the maximal condition for $\langle g\rangle$-invariant subgroups for each element $g \notin \boldsymbol{F i n}(G)$.

Theorem 30. Let $A$ be a vector space over the field $F$ and let $G$ be a finitely generated soluble subgroup of $G L(F, A)$ satisfying max-icd. Suppose that $G$ and its finitary radical both have infinite central dimension. Then $G$ contains a normal subgroup $L$ satisfying the following conditions:

(i) $G / L$ is abelian-by-finite;

(ii) $L$ has infinite central dimension and $L / L^{\prime}$ is not finitely generated;

(iii) $L \leqslant \boldsymbol{F i n}(G)$;

(iv) the subgroup $L$ satisfies the maximal condition for $\langle g\rangle$-invariant subgroups for each element $g \notin \boldsymbol{F i n}(G)$.

As a consequence we obtain a description of the periodic soluble groups satisfying the maximal condition on subgroups of infinite central dimension.

Theorem 31. Let $A$ be a vector space over the field $F$ of prime characteristic $p$ and let $G$ be an infinite periodic soluble subgroup of $G L(F, A)$ satisfying the condition max-icd. Suppose that $G$ has infinite central dimension. Then $G$ contains a normal subgroup $L$ satisfying the following conditions:

(i) $L$ has finite index in $G$;

(ii) $L=K Q$, where $K$ is a normal bounded nilpotent $p$-subgroup and $Q$ is a Prüfer $q$-group for some prime $q \neq p$;

(iii) $K$ has finite central dimension;

(iv) $A$ is $K$-nilpotent;

(v) $K$ has a finite $G$-composition series;

(vi) $Q$ has infinite central dimension.

In conclusion we observe that the study of infinite dimensional linear groups in which the family of subgroups having infinite central dimension and satisfying some classical finiteness condition has been carried out in 
various other cases. Our goal has been to give the general flavour of some of the results that can be obtained when certain finiteness conditions hold, so we presented only certain key situations. The interested reader can also find a number of papers related to these results, a list which includes, but is not limited to, the following papers: [4, 5, 8-10, 25-31] and [43].

\section{References}

[1] R. Baer, Endlichkeitskriterien für Kommutatorgruppen, Math. Ann. 124 (1952), 161-177.

[2] M. Bernkopf, A history of infinite matrices, Arch. History Exact Sci. 4 (1968), no. 4, 308-358, A study of denumerably infinite linear systems as the first step in the history of operators defined on function spaces.

[3] R. G. Cooke, Infinite matrices and sequence spaces, Dover Publications, Inc., New York, 1965.

[4] O. Yu Dashkova, M. R. Dixon, and L. A. Kurdachenko, Infinite dimensional linear groups with the restrictions on subgroups of infinite rank, Proceedings of Gomel University 3 (2006), 109-123.

[5] O. Yu Dashkova, M. R. Dixon, and L. A. Kurdachenko, Linear groups with rank restrictions on the subgroups of infinite central dimension, J. Pure Appl. Algebra 208 (2007), no. 3, 785-795.

[6] R. Dedekind, Über Gruppen deren sämtliche Teiler Normalteiler sind, Math. Ann. 48 (1897), 548-561.

[7] M. R. Dixon, M. J. Evans, and L. A. Kurdachenko, Linear groups with the minimal condition on subgroups of infinite central dimension, J. Algebra 277 (2004), 172186.

[8] M. R. Dixon and L. A. Kurdachenko, Linear groups with infinite central dimension, Groups St. Andrews 2005. Vol. 1, London Math. Soc. Lecture Note Ser., vol. 339, Cambridge Univ. Press, Cambridge, 2007, pp. 306-312.

[9] M. R. Dixon and L. A. Kurdachenko, Abstract and linear groups with some specific restrictions, Meeting on Group Theory and its applications, Madrid, Zaragoza 2011, 2012, pp. 87-106.

[10] M. R. Dixon, L. A. Kurdachenko, J. M. Muñoz-Escolano, and J. Otal, Trends in infinite dimensional linear groups, Groups St Andrews 2009 in Bath. Volume 1, London Math. Soc. Lecture Note Ser., vol. 387, Cambridge Univ. Press, Cambridge, 2011, pp. 271-282.

[11] M. R. Dixon, L. A. Kurdachenko, and J. Otal, Linear groups with bounded action, Algebra Colloq. 18 (2011), no. 3, 487-498.

[12] M. R. Dixon, L. A. Kurdachenko, and J. Otal, Linear groups with finite dimensional orbits, Ischia Group Theory 2010, World Sci. Publ., Hackensack, NJ, 2012, pp. 131145 .

[13] M. R. Dixon, L. A. Kurdachenko, and J. Otal, Linear analogues of theorems of Schur, Baer and Hall, Int. J. Group Theory 2 (2013), no. 1, 79-89. 
[14] M. R. Dixon, L. A. Kurdachenko, and J. Otal, On the structure of some infinite dimensional linear groups, Comm. Algebra 45 (2017), no. 1, 234-246.

[15] M. R. Dixon, L. A. Kurdachenko, and I. Ya. Subbotin, On an analogue of a theorem of $p$. hall for infinite dimensional linear groups, to appear.

[16] M. R. Dixon, L. A. Kurdachenko, and I. Ya. Subbotin, On composition factors in modules over some group rings, to appear.

[17] M. R. Dixon, L. A. Kurdachenko, and I. Ya. Subbotin, Ranks of Groups: The Tools, Characteristics, and Restrictions, John Wiley \& Sons, Inc., Hoboken, NJ, 2017.

[18] M. De Falco, F. de Giovanni, C. Musella, and Y. P. Sysak, On the upper central series of infinite groups, Proc. Amer. Math. Soc. 139 (2011), no. 2, 385-389.

[19] P. Hall, Finite-by-nilpotent groups, Proc. Cambridge Philos. Soc. 52 (1956), 611616.

[20] P. Hall and C. R. Kulatilaka, A property of locally finite groups, J. London Math. Soc. 39 (1964), 235-239.

[21] W. Holubowski, Algebraic properties of groups of infinite matrices, Monografia (Gliwice) [Monograph (Gliwice)], vol. 671, Wydawnictwo Politechniki Śląskiej, Gliwice, 2017, in Russian.

[22] M. I. Kargapolov, On a problem of O. Yu. Schmidt, Sibirsk. Mat. Z̆. 4 (1963), $232-235$.

[23] L. Kurdachenko and I. Subbotin, On some infinite dimensional linear groups, Groups St. Andrews 2001 in Oxford. Vol. II, London Math. Soc. Lecture Note Ser., vol. 305, Cambridge Univ. Press, Cambridge, 2003, pp. 377-384.

[24] L. A. Kurdachenko, Modules over group rings with some finiteness conditions, Ukrain. Mat. Zh. 54 (2002), no. 7, 931-940.

[25] L. A. Kurdachenko, On some infinite dimensional linear groups, Note Mat. 30 (2010), no. suppl. 1, 21-36.

[26] L. A. Kurdachenko, J. M. Muñoz-Escolano, and J. Otal, Antifinitary linear groups, Forum Math. 20 (2008), no. 1, 27-44.

[27] L. A. Kurdachenko, J. M. Muñoz-Escolano, and J. Otal, Locally nilpotent linear groups with the weak chain conditions on subgroups of infinite central dimension, Publ. Mat. 52 (2008), no. 1, 151-169.

[28] L. A. Kurdachenko, J. M. Muñoz-Escolano, and J. Otal, Soluble linear groups with some restrictions on subgroups of infinite central dimension, Ischia Group Theory 2008, World Sci. Publ., Hackensack, NJ, 2009, pp. 156-173.

[29] L. A. Kurdachenko, J. M. Muñoz-Escolano, and J. Otal, Groups acting on vector spaces with a large family of invariant subspaces, Linear Multilinear Algebra 60 (2012), no. 4, 487-498.

[30] L. A. Kurdachenko, J. M. Muñoz-Escolano, J. Otal, and N. N. Semko, Locally nilpotent linear groups with restrictions on their subgroups of infinite central dimension, Geom. Dedicata 138 (2009), 69-81.

[31] L. A. Kurdachenko, J. M. Muñoz-Escolano, J. Otal, and N. N. Semko, Locally nilpotent linear groups with some restrictions on subgroups of infinite central dimension, Dopov. Nats. Akad. Nauk Ukr. Mat. Prirodozn. Tekh. Nauki (2009), no. $3,25-28$. 
[32] L. A. Kurdachenko, J. Otal, and I. Ya. Subbotin, Groups with Prescribed Quotient Groups and Associated Module Theory, Series in Algebra, World Scientific, Singapore, 2002, Volume 8.

[33] L. A. Kurdachenko, N. N. Semko, and I. Ya. Subbotin, Insight into Modules over Integral Domains, Mathematics and its Applications, vol. 75, Proceedings of Institute of Mathematics, Kiev, Ukraine, Kiev, 2008.

[34] L. A. Kurdachenko and H. Smith, Groups in which all subgroups of infinite rank are subnormal, Glasg. Math. J. 46 (2004), no. 1, 83-89.

[35] L. A. Kurdachenko and I. Ya. Subbotin, On minimal Artinian modules and minimal Artinian linear groups, Int. J. Math. Math. Sci. 27 (2001), no. 12, 707-714.

[36] L. A. Kurdachenko and I. Ya. Subbotin, On some infinite-dimensional linear groups, Comm. Algebra 29 (2001), no. 2, 519-527.

[37] L. A. Kurdachenko and I. Ya. Subbotin, On some infinite dimensional linear groups, Southeast Asian Bull. Math. 26 (2003), no. 5, 773-787.

[38] L. A. Kurdachenko and I. Ya. Subbotin, Linear groups with the maximal condition on subgroups of infinite central dimension, Publ. Mat. 50 (2006), no. 1, 103-131.

[39] L. A. Kurdachenko. and I. Ya. Subbotin, A brief history of an important classical theorem, Adv. Group Theory Appl. 2 (2016), 121-124.

[40] A. G. Kurosh and S. N. Chernikov, Solvable and nilpotent groups, Uspehi Matem. Nauk (N.S.) 2 (1947), no. 3(19), 18-59.

[41] A. I. Maltsev, On groups of finite rank, Mat. Sbornik 22 (1948), 351-352.

[42] G. A. Miller and H. Moreno, Non-abelian groups in which every subgroup is abelian, Trans. Amer. Math. Soc. 4 (1903), 398-404.

[43] J. M. Muñoz-Escolano, J. Otal, and N. N. Semko, Periodic linear groups with the weak chain conditions on subgroups of infinite central dimension, Comm. Algebra 36 (2008), no. 2, 749-763.

[44] B. H. Neumann, Groups with finite classes of conjugate elements, Proc. London Math. Soc. (3) 1 (1951), 178-187.

[45] B. H. Neumann, Groups covered by permutable subsets, J. London Math. Soc. 29 (1954), 236-248.

[46] B. H. Neumann, Groups with finite classes of conjugate subgroups, Math. Z. 63 (1955), 76-96.

[47] A. Yu. Olshanskii, An infinite simple torsion-free Noetherian group, Izv. Akad. Nauk SSSR Ser. Mat. 43 (1979), no. 6, 1328-1393.

[48] A. Yu. Olshanskii, An infinite group with subgroups of prime orders, Izv. Akad. Nauk SSSR Ser. Mat. 44 (1980), no. 2, 309-321, 479.

[49] A. Yu Olshanskii, Groups of bounded exponent with subgroups of prime order, Algebra i Logika 21 (1982), 553-618 (Russian), English transl. in Algebra and Logic, 21 (1982), 369-418.

[50] R. E. Phillips, Finitary linear groups: a survey, Finite and Locally Finite Groups (Istanbul 1994) (B. Hartley, G. M. Seitz, A. V. Borovik, and R. M. Bryant, eds.), NATO Adv. Sci. Inst. Ser. C Math. Phys. Sci., vol. 471, Kluwer Acad. Publ., Dordrecht, 1995, pp. 111-146. 
[51] O. J. Schmidt, Groups all of whose subgroups are nilpotent, Mat. Sb. 31 (1924), 366-372 (Russian).

[52] D. I. Zaitsev, The existence of direct complements in groups with operators, Studies in Group Theory (Russian), Izdanie Inst. Mat. Akad. Nauk Ukrain. SSR, Kiev, 1976, pp. 26-44, 168.

[53] D. I. Zaitsev, Infinitely irreducible normal subgroups, Structures of groups and properties of their subgroups (Russian), Inst. Kibernet., Akad. Nauk Ukrain. SSR, Kiev, 1978, pp. 17-38, 150-151.

\section{CONTACT INFORMATION}

Martyn R. Dixon Department of Mathematics, University of Alabama, Tuscaloosa, AL 35487-0350, U.S.A. E-Mail(s): mdixon@ua.edu

Leonid. A.

Kurdachenko

Nikolaj N. Semko

Igor Ya. Subbotin
Department of Algebra, Faculty of Mathematics and Mechanics, National University of Dnepropetrovsk, Gagarin prospect 72, Dnipro 10, 49010, Ukraine E-Mail(s): Ikurdachenko@i.ua

Department of Mathematics, National State Tax Service Academy of Ukraine, 09200 Irpin, Ukraine

E-Mail(s): n-semko@mail.ru

Department of Mathematics and Natural Sciences, National University, 5245 Pacific Concourse Drive, Los Angeles, CA 90045-6904, U.S.A

E-Mail(s): isubboti@nu.edu

Received by the editors: 20.12.2019. 